\title{
Zum psychomotorischen Einsatz von Schwämmen und Wäscheklammern in der frühen Kindheit
}

\section{Warum Alltagsmaterialien?}

Eine psychomotorische Entwicklungsbegleitung mit Alltagsmaterialien ist in der sich verändernden frühkindlichen Lebensumwelt sehr sinnvoll. Die zunehmende Institutionalisierung von Kindheit und die Automatisierung ihrer Umwelt lassen fundamentale Erfahrungsräume schwinden, die den Aufbau von Ressourcen und die Entwicklung der Kinder fördern. Gerade weil Kinder der heutigen Zeit einer Vielzahl an vorgefertigtem Spielmaterial und einer extremen Reizüberflutung der Kommerzwelt gegenüberstehen, schafft der Einsatz von Alltagsmaterialien in der frühen Kindheit neue kreative Erfahrungsräume.

Ein weiterer positiver Nebeneffekt ist die kostengünstige Anschaffung von Altagsmaterialien, da eine Vielzahl an Materialien in der alltäglichen Umgebung bereits vorhanden oder kostengünstig zu erwerben ist. Die spielerischen Erfahrungen mit den Alltagsmaterialien, die in der Arbeit mit den Kindern gemacht werden, können durch die frühpädagogischen Fachkräfte darüber hinaus an die Eltern als zentrale Bezugspersonen weitergegeben werden, sodass auch diese Kenntnis darüber erwerben, mit alltäglichen Dingen, die das Kind umgeben, Spieleanlässe zu schaffen. Aber auch eine Einbindung der Eltern in das Sammeln von Alltagsmaterialien wäre denkbar. Oft sind es die einfachen, alltäglichen Dinge, mit denen sich die Kinder über Stunden beschäftigen können und die ihnen Freude bereiten.

\section{Ausgewählte Alltagsmaterialien}

Sowohl Schwämme als auch Wäscheklammern sollten als Alltagsmaterialien in einer unbestimmten, aber größeren Mengen zur Verfügung stehen. Ideal ist es, diese in verschiedenen Farben zu erwerben. Die beschriebenen Materialien gelten als sogenannte Basismaterialien, die um weitere Zusatzmaterialien ergänzt werden können, damit verschiedenste Erfahrungsmöglichkeiten für die Kinder entstehen. Die Kinder können somit auf vielfältige Weise ihre Bedürfnisse ausleben und sich nach und nach auf andere Reize einlassen. Der Fantasie sind dabei keine Grenzen gesetzt.

\section{Schwämme}

Das Alltagsmaterial Schwamm ist ein sehr weiches Material von relativ fester Konsistenz. Das Kind kann sich nicht daran verletzen. Deshalb ist es ein besonders gut geeignetes Material, um Kinder selbsttätig ihre Erfahrungen mit diesem sammeln zu lassen. Das Ma- terial kann sowohl zum Konstruieren als auch zum Toben genutzt werden. Schwämme gibt es in den verschiedensten Ausführungen. Es gibt sie in unterschiedlichen Farben und Größen, aber auch mit differenten Strukturen und Konsistenz. So sind die einen fein- oder grobporiger und die anderen von ihrer Konsistenz her weicher (zum Beispiel Naturschwamm) oder rauer (zum Beispiel Topfreiniger). Die Förderschwerpunkte dieses Materials liegen beim Werfen und Konstruieren, aber auch beim Balancieren (Köckenberger 2002, 193).

\section{Wäscheklammern}

Im Gegensatz zu dem Schwammmaterial, das die Grobmotorik der Kinder fördert, sind Wäscheklammern gut dazu geeignet, die feinmotorische Koordination zu trainieren. Wäscheklammern sind von harter und relativ robuster Konsistenz. Die Verletzungsgefahr liegt hier deutlich höher und bedarf deshalb der ständigen Beobachtung beziehungsweise Begleitung durch einen Erwachsenen. Der Umgang mit den Wäscheklammern erfordert Achtsamkeit, Geschicklichkeit und Kraft der Kinder. Wäscheklammern werden aus unterschiedlichen Materialien hergestellt, es gibt sie zum Beispiel aus Holz oder Kunststoff. Sie variieren aber auch in ihrer Form und Stabilität. För- 
derschwerpunkte dieses Materials sind die Feinmotorik, taktile Wahrnehmung und Achtsamkeit (Köckenberger 2002, 249).

\section{Beispiele für die praktische Umsetzung}

\section{Praxisbeispiel 1: Waschstraße - Basismaterial: Schwämme}

Zusätzlich zu den Schwämmen werden ein Rollbrett und Klebeband benötigt (ggf. Seile, Wasser, große Schaumstoffteile, weiche Bürsten).

Vorbereitung: Mithilfe des Klebebands wird eine Waschstraße abgeklebt. Durch diese Straße soll das Rollbrett geschoben werden können. Entlang der Waschstraße werden verschiedenste Materialien ausgelegt, an denen sich die Kinder frei bedienen können.

Aufgabe: Es wird von den Kindern eine Waschstraße gebildet. Eines der Kinder wird ausgewählt, das sich auf das Rollbrett legt. Anschließend wird dieses beim Vorbeifahren von den anderen Kindern gewaschen.

Erfahrungswert: Die Waschstraße setzt der Fantasie der Kinder keine Grenzen, wenn verschiedene Materialien zur Konstruktion einer Waschstraße zur Verfügung stehen. Sie kann sowohl im Innenbereich als auch auf dem Außengelände, beispielsweise im Sommer mit Wasser, gespielt werden. Zuletzt Genanntes hat den Vorteil, dass die Kinder leichter bekleidet sein können und die taktile Wahrnehmung umso intensiver gefördert wird. Den Kindern werden verschiedene Erfahrungen ermöglicht, beispielsweise Körperwahrnehmung, taktile Reize, Konstruktions- und physikalische Erfahrungen (Abb.1).

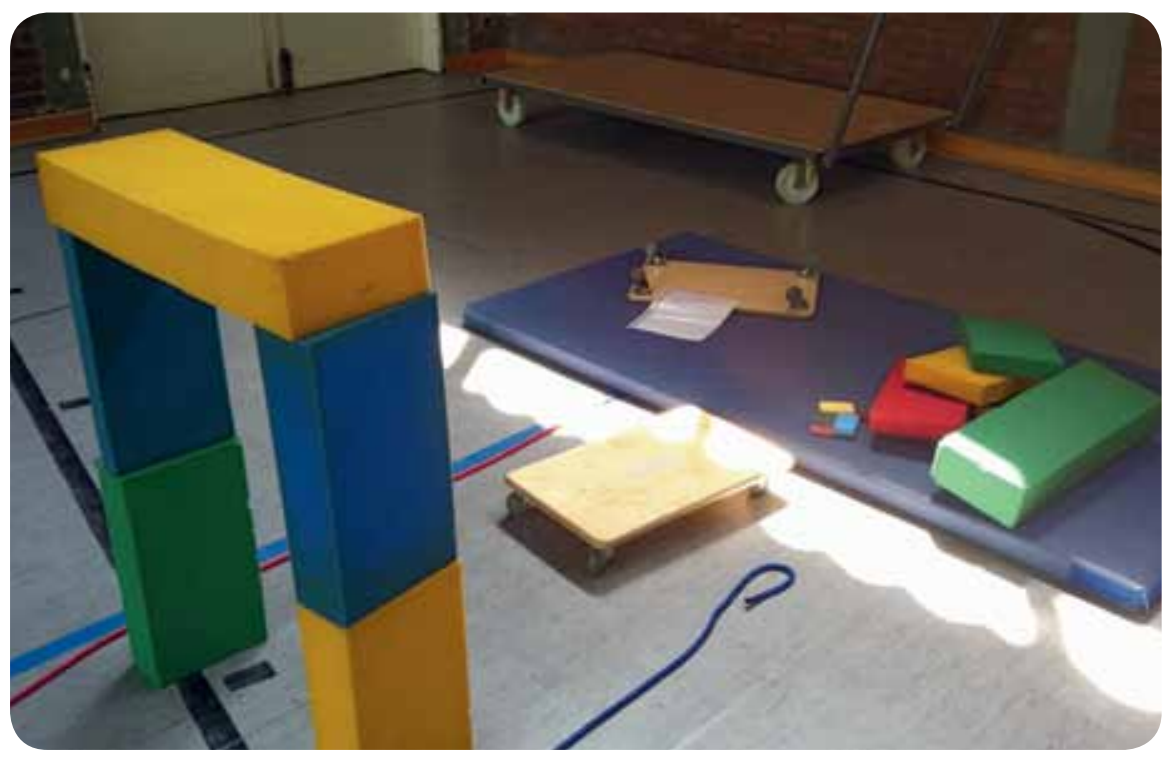

Abb. 1: Eine Waschstraßenkonstruktion mithilfe von Seilen und Schaumstoffteilen

\section{Praxisbeispiel 2: Stempelbild - Basismaterial: Schwämme}

Als Zusatzmaterialien neben den Schwämmen werden Papier, Wasserfarbe und ein Behälter mit Wasser benötigt.

Vorbereitung: Gegebenenfalls Kleidungs- und Unterlagenschutz bereitstellen.

Aufgabe: Mithilfe von verschiedenen Schwämmen (fein- und grobporig) und der Wasserfarbe sollen die Kinder ein Kunstwerk anfertigen. Dabei sollen möglichst verschiedene Handhabungstechniken wie Wischen und Stempeln erforscht werden.

Erfahrungswert: Die Stempelbilder ermöglichen eine kreative Umfunktionierung des Materials. Im Alltag wird der Schwamm in der Regel zum Entfernen von Substanzen verwandt, während er hier als Pinselersatz zum Anbringen von Substanzen genutzt wird. Kinder unter dem dritten Lebensjahr beherrschen die Pinselarbeit, welche innen eine feinmotorische Fingertechnik und Handbewegung abverlangt, noch nicht; dies wird dabei auch nicht

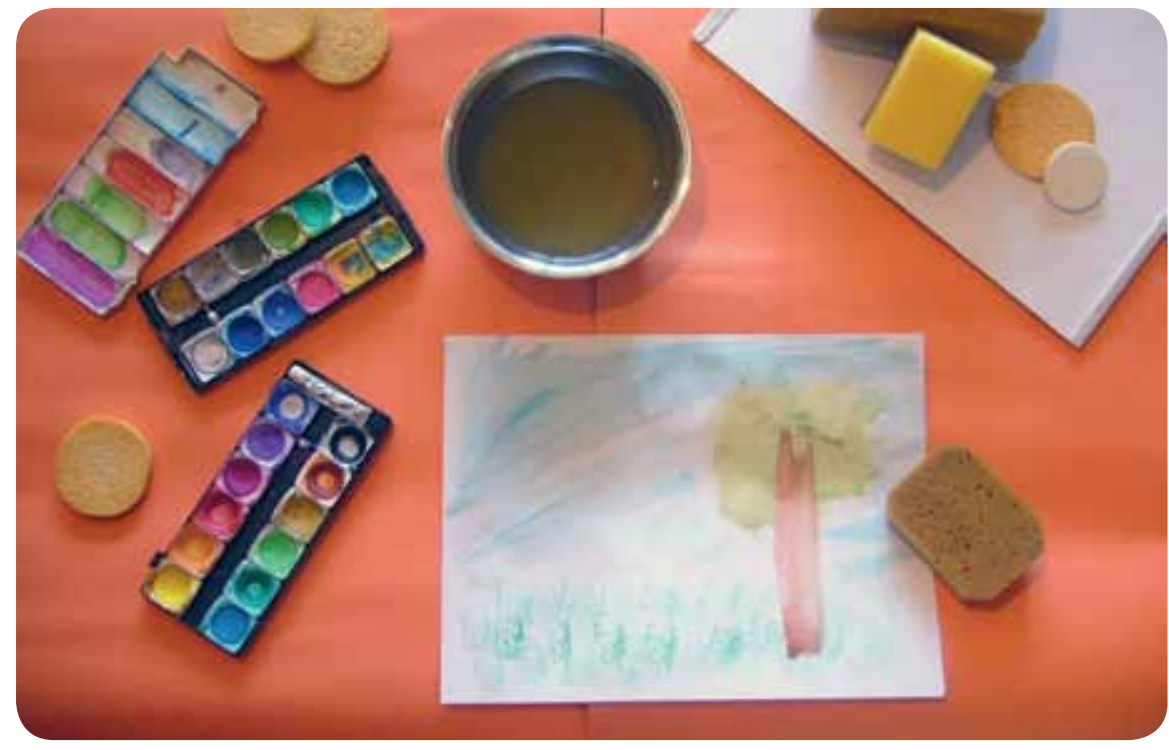

Abb. 2: Stille und kreative Arbeit beim Experimentieren mit dem Schwamm 
zu einer Notwendigkeit. Stempelbilder können auch mit anderen Materialien wie beispielsweise Korken angefertigt werden. Die Kinder erlangen dabei besonders kreative und ästhetische Erfahrungen (Abb.2).

\section{Praxisbeispiel 3: Greifarm - Basismaterial: Wäscheklammern}

Neben den Wäscheklammern werden zwei Gefäße benötigt (am besten flache Schalen, da hohe Gefäße mit glattem Rand das Aufnehmen der Gegenstände erschweren) sowie verschiedene Alltagsgegenstände.

Vorbereitung: Verschiedene Alltagsgegenstände in ein Gefäß geben, ein weiteres leeres Gefäß danebenstellen.

Aufgabe: Mithilfe von ein bis zwei Wäscheklammern soll versucht werden, verschiedene Alltagsgegenstände aufzunehmen und in das andere Gefäß abzulegen.

Erfahrungswert: Beim Greifarm findet eine Übertragung des sogenannten Pinzetten-Griffes mithilfe der Wäscheklammern statt. Von den Kindern wird eine feinmotorische und kreative Arbeit verlangt. Durch Ausprobieren müssen neue Möglichkeiten herausgefunden werden, Gegenstände mithilfe des Pinzetten-Griffes zu erfassen und transportfähig zu machen. Dabei erhalten sie die Möglichkeit, Erfahrungen mit Teamarbeit zu machen, zum Beispiel indem sie fein koordinierte $\mathrm{Be}$ wegungen mit einem Partner abstimmen und Arbeitsteilung vornehmen. Es konnte beobachtet werden, dass zuvor sortierte Gegenstände aus der einen Schale, nachdem sie in die andere Schale abgelegt wurden, wieder zurück in die ursprüngliche Schale transportiert und an denselben Platz zurückgelegt wurden. Dem ordnungsgemäßen

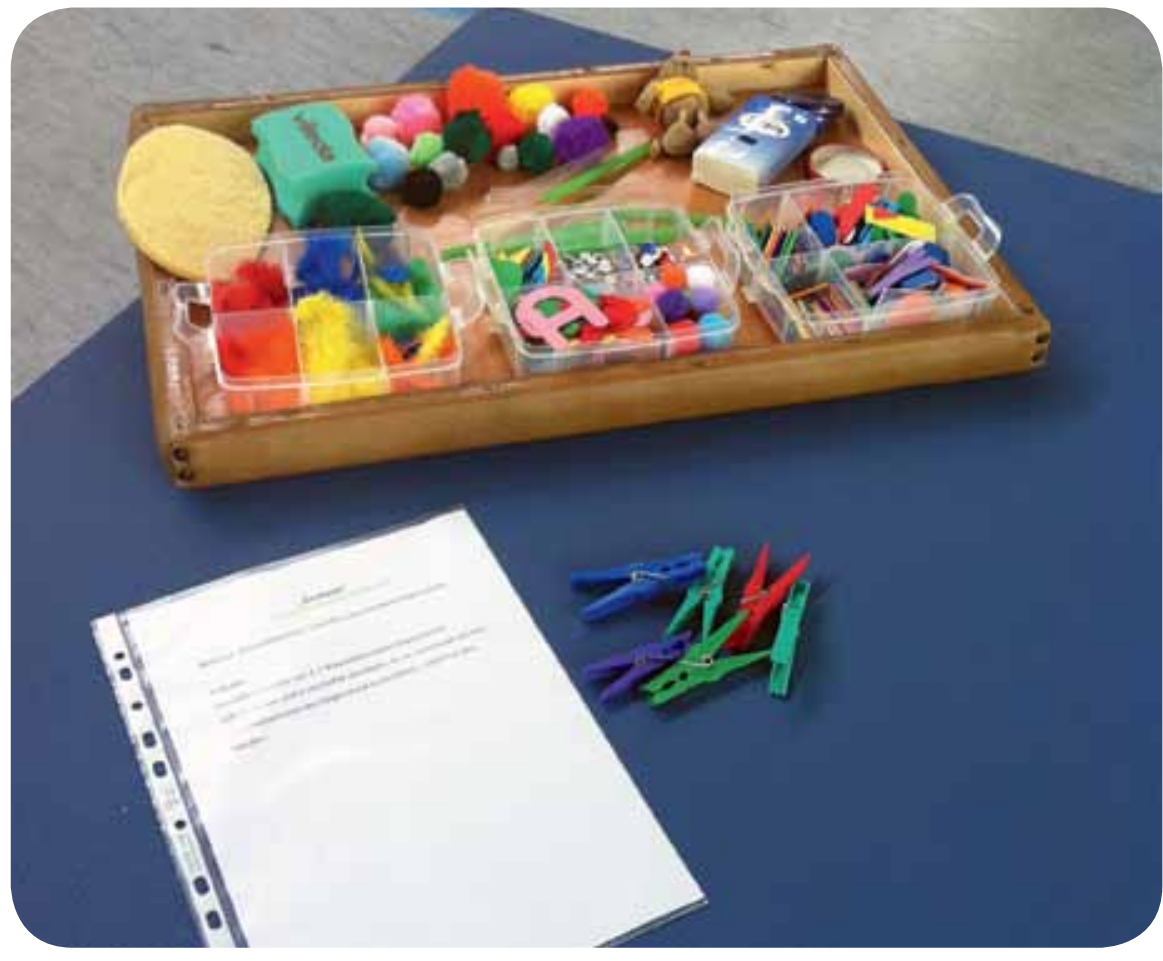

Abb. 3: Eine exemplarische Sammlung von Alltagsmaterialien zur Ausübung des Praxisbeispiels Greifarm

Zurücksortieren scheint eine starke innere Motivation zugrunde gelegt zu sein; es macht ein Verlangen nach Ordnung sichtbar (Montessori $200981 \mathrm{ff}$ ) (Abb. 3).

\section{Fazit}

Die drei ausgewählten Praxisbeispiele können nur einen sehr kleinen Bereich von vielfältigen Erfahrungsmöglichkeiten aufzeigen, die mithilfe der Alltagsmaterialien Schwamm und Wäscheklammer gemacht werden können. Diese können sowohl eine vertiefte stille Arbeit - ein Bei-sich-Sein - der Kinder als auch soziale Kompetenzen unter Gleichaltrigen fördern. An dieser Stelle soll aber noch einmal ganz besonders darauf verwiesen werden, dass die Kinder auch ohne Aufgaben dazu in der Lage sind, ihre Erfahrungen selbstwirksam mit den zur Verfügung gestellten Materialien zu sammeln. Die aufgezeigten Praxisbeispiele dienen zur Orientierung, Motivierung und vertieften beziehungsweise spezifischen
Förderung und Forderung der Kinder. Es gilt die Regel: Weniger ist mehr. Für das Sammeln von neuen Erfahrungen ist es sinnvoll, zunächst einzelne Materialien zur Verfügung zu stellen, um die Kinder nicht zu überfordern. Die Komponente Zeit spielt ebenfalls eine ganz besondere Rolle. Den Kindern sollte ermöglicht werden, ohne Zeitdruck und formale Vorgaben ihre Erfahrungen zu sammeln, frei nach dem Motto: Die Kinder machen lassen und ihrer Fantasie keine Grenzen setzen.

\section{(II Literatur}

Köckenberger, H. (2002): Bewegungsspiele mit Alltagsmaterial. 3. Aufl. borgmann, Dortmund

Montessori, M. (2009): Kinder sind anders. 14. Aufl. Klett-Cotta. Stuttgart

\section{Christina Hartmann}

DOI 10.2378/ motorik2014.art31d 